\title{
Belphégor
}

\section{La novellisation dans le réseau intermédiatique du Petit Nicolas}

\section{Benoît Glaude}

\section{Q OpenEdition}

1 Journals

\section{Electronic version}

URL: https://journals.openedition.org/belphegor/1074

DOI: $10.4000 /$ belphegor.1074

ISSN: 1499-7185

\section{Publisher}

LPCM

\section{Electronic reference}

Benoît Glaude, "La novellisation dans le réseau intermédiatique du Petit Nicolas", Belphégor [Online], 16-1 | 2018, Online since 16 July 2018, connection on 19 October 2021. URL: http:// journals.openedition.org/belphegor/1074 ; DOI: https://doi.org/10.4000/belphegor.1074

This text was automatically generated on 19 October 2021

\section{(c) (i) (9)}

Belphégor est mis à disposition selon les termes de la Licence Creative Commons Attribution - Pas d'Utilisation Commerciale - Pas de Modification 4.0 International. 


\title{
La novellisation dans le réseau intermédiatique du Petit Nicolas
}

\author{
Benoît Glaude
}

1 C'est presque un lieu commun de déplorer que les études francophones d'adaptations littéraires en bande dessinée peinent à sortir du cadre de la question de fidélité. Il est vrai qu'elles le font avec un temps de retard sur la recherche anglophone. Cependant, ce qui continue à poser problème est moins la révision du principe de fidélité ${ }^{1}$ que le dépassement d'une conception téléologique du processus adaptatif. Le recours automatique aux concepts d'œuvres source et cible trahit une conception de l'adaptation comme traduction à sens unique, donc "en termes de légitimation culturelle, le système le plus faible (la bande dessinée) s'appuyant sur le système le plus fort (la littérature) $»^{2}$. Cet article remet en question trois postulats dans le traitement habituel du sujet, selon lesquels l'adaptation littéraire se ferait à sens unique, elle ne constituerait pas un processus de création originale et elle s'attaquerait à un modèle indépassable. Plus précisément, il s'agira d'aborder la BD non comme cible mais comme source d'adaptation littéraire, de souligner la parenté entre les processus créatifs d'adaptation et d'autoadaptation, et d'envisager que le fruit d'une telle création puisse être médiagénique. La médiagénie désigne l'adéquation fusionnelle d'un récit et d'un média ${ }^{3}$, qui le rendrait temporairement réfractaire à toute adaptation, même si le temps finit souvent par invalider les arguments de l'inadaptabilitét. Si l'on accepte l'idée qu'un récit de BD puisse devenir une œuvre littéraire, pourquoi ne pas envisager qu'il se retrouve, au moins pour un temps, en parfaite adéquation avec le média littéraire?

2 La série du Petit Nicolas offre un corpus idéal pour tester ces hypothèses. Au cours d'une première phase d'autoadaptations (1954-1965) assurées par Jean-Jacques Sempé et René Goscinny, elle a transité du cartoon - dessin d'humour isolé ou séquentialisé, qui « comporte à la fois caricature, schématisation et satire $»^{5}$ - à la bande dessinée, pour aboutir aux nouvelles illustrées devenues des classiques de la littérature enfantine. La série se trouve actuellement dans une nouvelle phase d'adaptations (2002-2018), sans la collaboration de ses créateurs, où, après avoir été transposée sur grand et petit écran, 
en films live-action et en dessins animés, elle continue de se décliner en de multiples produits dérivés, dont plusieurs séries de novellisations. À ce jour, ce réseau d'adaptations intermédiatiques comporte plus de deux cents nouvelles et romans. Certaines de ces œuvres - pas toutes - constituent des novellisations, c'est-à-dire des transpositions littéraires de cartoons, bandes dessinées ou dessins animés. Je propose ici de relire quelques nouvelles du Petit Nicolas comme des novellisations directes - et quelques romans, comme des novellisations indirectes - des BD parues en 1955-1956, en considérant leur transmédiatisation à la fois comme une pratique culturelle et comme un processus créatif.

\section{L'autoadaptation comme pratique culturelle}

3 À l'automne 2017, les éditions IMAV publient une «bande dessinée originale » du Petit Nicolas $^{6}$, antérieure aux célèbres nouvelles, parue dans le magazine belge Moustique au milieu des années 1950. Dans les années 2000, cet éditeur avait déjà éveillé la curiosité pour la génétique des textes illustrés de Sempé et Goscinny, en republiant cent trentecinq nouvelles restées inédites en recueils. Les grandes lignes de leur histoire éditoriale sont connues. Henri Amouroux, qui dirigeait le supplément dominical du quotidien girondin Sud Ouest (où le Bordelais Sempé avait fait ses débuts de dessinateur de presse), y publia en mars 1959 la première d'une longue série de nouvelles hebdomadaires. Ainsi, Sud Ouest Dimanche eut la primeur des deux cents et quelques nouvelles illustrées du Petit Nicolas, dont une majorité reparurent dans l'hebdomadaire Pilote, dès le premier numéro en octobre 1959. De 1960 à 1964, les auteurs en publièrent un recueil par an chez Denoël, compilant moins de la moitié de leurs nouvelles, après quoi ils mirent un terme à leur collaboration. Leurs souvenirs discordants de cette interruption s'entendent au moins sur le principe de ne pas lasser les lecteurs? ${ }^{7}$.

4 L'historiographie des nouvelles illustrée, c'est-à-dire de la forme sous laquelle le Petit Nicolas a passé à la postérité, privilégie la période 1959-1965. Or, l'œuvre a connu une existence antérieure et postérieure. Pour étudier cet amont et cet aval de son histoire, je propose de les décrire comme des processus adaptatifs. Dès la première heure, les lecteurs jouèrent un rôle crucial dans la constitution de ce réseau intermédiatique, même s'ils devaient probablement percevoir ces multiples déclinaisons davantage comme des continuations que comme des adaptations. En effet, l'œuvre est née dans des périodiques diffusés à une échelle régionale (sauf Pilote), organes de presse aux moyens éditoriaux nuls ou limités. Pendant cinquante ans, les lecteurs ne disposaient pas d'une édition exhaustive, qui eût permis des recoupements, ils attendaient simplement la suite des histoires du Petit Nicolas. Après avoir reconstitué ce corpus disparate, je ne me contenterai pas de décrire (au point suivant) une telle adaptation comme une mise en réseau intermédiatique, mais aussi (dans ce point) comme l'expérience d'une pratique culturelle. Pour Jan Baetens, «l'auto-adaptation, loin de relever des seules considérations stratégiques de l'artiste, [...] rencontre aussi les exigences du public, qui loin d'attendre ce type d'auto-autoadaptation, se met à les réclamer $»^{8}$. Cette tendance commençait à poindre dans les réactions épistolaires de la communauté des lecteurs du Moustique.

5 Le magazine généraliste édité par les frères Dupuis (comme, à l'époque, les périodiques Bonnes soirées, Spirou et bientôt Risque-Tout) fêta en novembre 1954 ses trente années d'existence sur le marché belge francophone, à côté de son pendant flamand: 
Humoradio. Pendant trente ans, l'hebdomadaire avait perpétué une formule emblématisée par son dessin humoristique en couverture, confié aux grands noms du journal de Spirou, alors qu'il diffusait un contenu plutôt sérieux, centré sur les programmes radiophoniques. Sans être aussi structurée que le club des Amis de Spirou, la communauté des lecteurs du Moustique s'exprimait en plusieurs endroits du magazine, non seulement sur les choix rédactionnels, mais également sur des questions personnelles. Le jeune rédacteur en chef René Henoumont perpétuait le principe, instauré par son aîné Jean Doisy, de se référer au «baromètre » des lecteurs".

Henoumont débuta au Moustique en lançant une nouvelle formule, en 1954, amorçant une rénovation qui aboutirait, en 1958, au TV Radio Moustique. S'il travaillait pour le compte des frères Dupuis, le rédacteur en chef renouvela le magazine sous l'aile de l'agence World Press, avec l'aide de son directeur Georges Troisfontaines et de plusieurs de ses employés, dont René Goscinny et Jean-Michel Charlier. L'agence bruxelloise, qui disposait également d'un bureau à Paris, plaçait des contenus publicitaires et rédactionnels, y compris des illustrations et des bandes dessinées, dans les magazines des éditions Dupuis. En outre, elle gérait la rédaction du magazine féminin Bonnes soirées et briguait celle du Moustique. Tout en restant aux mains des Dupuis, cette dernière déménagea de Charleroi à Bruxelles, dans le même bâtiment que la World Press. La nouvelle formule du magazine accordait plus de place à l'image, avec un cahier de seize pages de couleurs en héliogravure, sur un meilleur papier, en se référant au modèle français de Paris Match. Le Moustique proposa dorénavant un récit policier ou western par semaine - cette rubrique inaugurée par Georges Simenon serait alimentée par René Goscinny souvent sous pseudonymes (René Macaire, René Maldecq) -, ainsi que des portraits de vedettes de la chanson, du spectacle, du cinéma, et il commença à publier les programmes télévisés à côté des programmes radiophoniques.

7 Les couvertures photographiques alternèrent progressivement avec les couvertures dessinées, qui ne seraient plus qu'exceptionnelles à partir de 1959. Parmi les illustrateurs qui continuèrent à en produire durant cette période de transition, se trouve Jean-Jacques Sempé, dont Charles Dupuis avait prédit, dès 1954, qu'il égalerait son dessinateur fétiche, André Franquin ${ }^{10}$. Le courrier des lecteurs lui était également favorable :

Bravo pour Sempé! Je ne sais si d'autres lecteurs vous ont déjà félicités pour le bon goût des caricatures de Sempé. Nous sommes un groupe de jeunes qui attendons chaque jeudi la sortie de "Moustique » pour nous divertir et commenter ensemble vos dessins humoristiques. ${ }^{11}$

De 1952 à 1958, l'illustrateur français y publia de nombreux cartoons et près de soixantedix couvertures reprenant généralement «en couleurs des travaux qui étaient déjà parus ailleurs $»^{12}$. Cependant, douze couvertures inédites ${ }^{13}$ mirent en scène un personnage créé pour Le Moustique, apparu sur l'une d'entre elles en janvier 1954 ( $\left.\mathrm{n}^{\circ} 1461\right)$, puis baptisé «le Petit Nicolas» dans un cartoon paru six mois plus tard ( $\left.n^{\circ} 1481\right)$. Le héros reparut régulièrement, dans des dessins réunis en quatrième de couverture ou incongrûment placés dans le récit policier hebdomadaire, de sorte que le Petit Nicolas de Sempé se retrouva au cœur des nouvelles policières de Goscinny. Les deux hommes ne se rencontrèrent qu'au printemps 1955, dans les bureaux parisiens de l'agence World Press.

9 Le personnage connut donc une première vie d'errance dans le magazine, déclinée en dessins autonomes - soit onze couvertures ${ }^{14}$ et trente-huit cartoons dus au seul Sempé - 
du 13 juin 1954 ( $\left.n^{\circ} 1481\right)$ au 2 octobre 1955 ( $\left.n^{\circ} 1549\right)$. Assez tôt, un lecteur réclama une pagination fixe pour « [s]on ami, le petit Nicolas » :

En quelques semaines, le petit Nicolas est devenu mon ami. Dès que j'ouvre mon "Moustique », c'est pour chercher ce sympathique garçon dont les facéties me ravissent. Une seule remarque : ne pourriez-vous le passer à la même page dans chaque numéro ${ }^{15}$

Le rédacteur en chef imagina de sérialiser le personnage, qui deviendrait une mascotte $\mathrm{du}$ Moustique ${ }^{16}$, sous la forme de gags en une planche qui auraient les honneurs du dos en couleurs du magazine ${ }^{17}$. Sempé cosigna la reprise de son personnage avec le scénariste Agostini, pseudonyme de Goscinny. Du 25 septembre $1955\left(n^{\circ} 1548\right)$ au 20 mai 1956 ( $\left.\mathrm{n}^{\circ} 1582\right)$, il connut donc une deuxième vie, en vingt-huit gags de $\mathrm{BD}$, plus un dernier le 11 mai 1958 ( $n^{\circ}$ 1685). Contribuant à la nouvelle formule du Moustique, le personnage sérialisé en bande dessinée avait « fait déjà parler de lui " ${ }^{18}$ en 1955 , comme Henoumont le faisait remarquer aux lecteurs de son magazine. Leur courrier s'en était fait l'écho :

Des apports récents, je dois dire que, de loin, le petit Nicolas l'emporte. C'est là un avis personnel, mais je tenais à vous le signaler. Je suis grand lecteur de journaux et fervent amateur de blagues dessinées. À ma connaissance, c'est la première fois qu'un dessinateur parvient à camper un personnage de gosse qui soit drôle sans être méchant. "Moustique », l'hebdomadaire de la famille, a réussi avec Sempé et Agostini cette gageure. Bravo, et longue vie au petit Nicolas !19

11 En dépit de ce succès annoncé, le personnage et son scénariste disparurent du magazine, fin mai 1956. L'illustrateur y poursuivit sa collaboration pendant deux ans et demi, mais sans reprendre nommément le Petit Nicolas. Plusieurs couvertures de divers dessinateurs le ressuscitèrent sans le nommer, ce qui indique qu'elles constituaient un patrimoine graphique commun, dont les idées pouvaient être recyclées. Propriété de Sempé, le personnage ne devint jamais une figure tutélaire du magazine, tel que René Henoumont l'avait envisagé, mais son aspect de petit garçon ordinaire permit plusieurs réappropriations ponctuelles ${ }^{20}$. En dehors de ces discrètes réminiscences, aucune réaction de lecteur ne fut publiée après l'arrêt inopiné de la série, qu'aucun rédactionnel n'annonça ni ne commenta non plus. Selon Goscinny, « nous avons fait trente pages avec "Nicolas" qui ont eu un succès fou en Belgique. C'est à ce moment-là que je me suis fait virer par Troisfontaines : notre bande en est restée là. ${ }^{21}$ En 1956, avec deux collègues de la World Press, le scénariste tenta de syndiquer la profession des auteurs de BD franco-belges pour défendre leurs intérêts face aux éditeurs et aux agences. Leur employeur, Georges Troisfontaines, les licencia tous les trois aussitôt qu'il eut vent de leur projet. Ne pouvant plus travailler pour Moustique, René Goscinny dut cesser de scénariser le Petit Nicolas, et Jean-Jacques Sempé jeta le gant ${ }^{22}$.

12 L'évolution de l'œuvre dans Moustique illustre une pratique culturelle relativement conflictuelle de l'autoadaptation, puisque la demande lectorale semble être entrée en contradiction avec les postures auctoriales des producteurs. Leurs témoignages tardifs, exprimés à plus de vingt ans de distance, sont toutefois sujets à caution. Selon Sempé, il s'agissait d'un travail de commande, imposé sans égard pour ses intentions artistiques : « ils m'ont demandé de le transformer en bandes dessinées, mais je ne voulais pas faire une bande dessinée, ce n'était pas mon truc du tout $»^{23}$. Il insiste sur sa vocation pour le dessin de presse, qu'il définit par opposition avec la BD.

Je crois qu'il s'agit d'une discipline [la $\mathrm{BD}$ ] très éloignée de la mienne. Je cherche à condenser, alors que la bande dessinée allonge et joue sur la durée. [...] Ce qui me plaît aussi, c'est de me dire : ce dessin-là, on ne pourrait pas le raconter, ni en faire 
un film ou quoi que ce soit d'autre, c'est vraiment du dessin humoristique, dans ce qu'il a de plus spécifique. ${ }^{24}$

Quant à René Goscinny, il employait plusieurs pseudonymes lorsqu'il collaborait au Moustique, pour la World Press. Trois ans après son licenciement, à la tête de sa propre agence et s'apprêtant à diriger l'hebdomadaire Pilote, il signa de son nom la reprise littéraire du Petit Nicolas dans Sud Ouest Dimanche. Cette nouvelle posture d'auteur ne se réduisait pas à une question contractuelle. Selon Sempé, Goscinny saisit une occasion d'assumer pleinement une posture littéraire, d'auteur au sens moderne : «J'avais déjà lu des textes de René. Mais son premier texte écrit en toute liberté est Le Petit Nicolas. $\aleph^{25}$ Même si elles ont été prépubliées dans des magazines dépourvus de prétention littéraire, les nouvelles recueillies chez l'éditeur littéraire Denoël renouaient avec le livre, dans un format carré qui évoquait aussi l'album. La création était bien sûr collective et actée comme telle par une répartition égale des droits d'auteurs. Cependant, si l'illustrateur avait été le créateur et le propriétaire du personnage, l'écrivain assumait seul ses choix d'écriture :

En fait, on œuvrait chacun dans notre coin, révèle Sempé. Je recevais ses feuillets tapés à la machine, sans la moindre rature. Une fois, seulement, je m'étais permis une suggestion et je l'ai regretté. Il faisait dire à l'un des enfants: "Nos mamans sont chouettes ". J'avais objecté qu'un enfant aurait plutôt dit: "Nos mères sont chouettes ». René, qui était ultrasensible, l'a mal pris. Je n'ai plus jamais fait la moindre remarque. ${ }^{26}$

\section{L'autoadaptation comme processus de création}

Le tableau suivant (fig. 1) établit des correspondances possibles entre les dessins et bandes dessinées parus dans Moustique (1954-1956), les nouvelles parues dans Pilote (1959-1965) et les novellisations de la récente série d'animation télévisée. Il existe aujourd'hui dans le catalogue Gallimard Jeunesse deux séries de novellisations des dessins animés de treize minutes ${ }^{27}$ : la première, signée par Valérie Latour-Burney, comporte sept novellisations d'une centaine de pages (2012-2015) destinées aux 8-12 ans, tandis que la seconde, publiée depuis 2012 sous le nom d'Emmanuelle Lepetit, compte à ce jour trente-huit titres d'une trentaine de pages destinés aux 6-8 ans. Douze des vingt-neuf gags de BD apparaissent en gras dans le tableau car ils constituent les hypotextes de novellisations les plus manifestes, en raison d'une similarité forte des intrigues. Les autres n'entretiennent qu'une parenté d'intrigue partielle, voire un simple rapport formel ou thématique, avec une ou plusieurs nouvelles. Dans la suite, les différents recueils seront abrégés comme suit ${ }^{28}: \mathrm{LPN}=$ Le Petit Nicolas; RPN $=$ Les Récrés $d u$ Petit Nicolas $;$ VPN = Les Vacances du Petit Nicolas ; JAE = Joachim a des ennuis; HI1-HI2 = Histoires inédites du Petit Nicolas, volume 1 ou 2. 


\begin{tabular}{|c|c|c|c|c|c|c|}
\hline \multicolumn{2}{|c|}{ Gags de BD } & Dessins & \multicolumn{3}{|r|}{ Nouvelles } & \multirow{2}{*}{$\begin{array}{c}\text { Romans } \\
\text { Titre }\end{array}$} \\
\hline $\mathbf{N}^{\circ}$ & & oustique & Pilote & Recueil & Titre & \\
\hline 1 & 1548 & 1496,1510 & 10 & HI1 & La trompette & En avant, la musique! \\
\hline 2 & 1549 & & 82 & HI1 & Les nouveaux voisins & \\
\hline 3 & 1550 & & 125 & HI1 & Flatch flatch & \\
\hline 4 & 1552 & 1500 & & HI1 & Mots croisés & \\
\hline 5 & 1551 & & 44 & VPN & La plage, c'est chouette & \\
\hline 6 & 1553 & 1514 & 77 & HI2 & Les ouvriers & \\
\hline 7 & 1554 & & 32 & LPN & Le vélo & Papa m'offre un vélo \\
\hline 8 & 1555 & 1528,1582 & 122 & HI2 & Le vitrier & \\
\hline 9 & 1556 & & 21 & HI2 & Chez le coiffeur & \\
\hline 10 & 1557 & & 13 & HI2 & Nicolas et Blédurt & \\
\hline 11 & 1558 & 1509,1663 & 33 & HI1 & Tuuuuut! & Papa casse mes jouets ! \\
\hline \multirow{2}{*}{12} & \multirow{2}{*}{1559} & & 92 & HI1 & Au chocolat et à la fraise & \\
\hline & & & 69 & RPN & Le vase rose du salon & \\
\hline 13 & 1560 & & 26 & LPN & Les cow-boys & La Bande des pirates \\
\hline [14] & 1561 & 1507 & 194 & $J A E$ & La craie & \\
\hline \multirow{2}{*}{16} & \multirow{2}{*}{1563} & \multirow{2}{*}{1528} & 108 & HI1 & Pamplemousse & \\
\hline & & & 6 & HI2 & Je fais des courses & \\
\hline 17 & 1564 & 1541 & 128 & HI1 & La fête foraine & \\
\hline 18 & 1565 & & 309 & HI1 & On va rentrer & Oh, la honte! \\
\hline 22 & 1569 & & 4 & LPN & Je suis malade & Adieu les mauvaises notes \\
\hline 23 & 1573 & & 19 & HI1 & La maison de Geoffroy & \\
\hline 24 & 1575 & & 72 & HI1 & Le croquet & \\
\hline 25 & 1576 & 1541 & 1 & HI1 & La séance de cinéma & \\
\hline 28 & 1582 & 1582 & 8 & IPN & Un souvenir qu'on va chérir & La photo de classe \\
\hline [29] & 1685 & 1493 & & & & \\
\hline
\end{tabular}

Fig. 1 : Quelques correspondances possibles entre les dessins et BD parus dans Moustique (1954-1956), les nouvelles parues dans Pilote (1959-1965) et les novellisations (2012-2017) du Petit Nicolas.

15 Les cas de parenté la plus ténue sont ceux qui montrent la limite de la subjectivité dans l'établissement de telles connexions intermédiatiques. L'intrigue du dix-huitième gag ${ }^{29}$, par exemple, où Nicolas, amené de force par sa mère au magasin de chaussures, essaye des dizaines de paires sans arrêter un choix, est résumée au début de la nouvelle « On va rentrer " (HI1). Cette brève relation modifie le dénouement de l'anecdote, devenue secondaire : la mère impose son choix d'une paire de souliers, qui ne convient pas aux pieds de son fils. Ce nouveau dénouement sert de point de départ au dessin animé Les Chaussures vertes, qui suit pour le reste la nouvelle «Je me cire» (HI2). Dans les trois versions du récit, le héros déteste par principe les chaussures qui lui sont proposées ou imposées, mais ce n'est que dans le dessin animé - qui sera novellisé sous le titre Oh, la honte! - qu'il s'inquiète de la réaction de ses camarades de classe. Cet ajout d'un sentiment de honte, qui vise probablement à rendre son refus plus vraisemblable, produit un effet contraire, en dénaturant son portrait psychologique. Dans les nouvelles de Sempé et Goscinny, le héros âgé de 6 à 10 ans $^{30}$ découvre à peine ce sentiment inculqué. Ainsi, dans "Le gros mot " (HI1), le père de Nicolas le force à reconnaitre qu'il a honte d'avoir proféré un juron, sans pourtant parvenir à lui faire comprendre en quoi jurer peut être honteux. Dans les illustrations de cette nouvelle, des bulles de paroles contiennent le mot prohibé, mais sous une pancarte qui le censure, autant qu'il l'est dans le texte littéraire ${ }^{31}$. Le clin d'œil à la bande dessinée est souligné par cet emploi plutôt emblématique qu'énonciatif de la bulle. On observe ainsi une rémanence de la $\mathrm{BD}$ dans l'illustration de roman, laquelle a constitué depuis le XIX ${ }^{\mathrm{e}}$ siècle - ainsi que le dessin de presse - l'une des nombreuses séries culturelles constitutives du média bande dessinée ${ }^{32}$. La plupart des cas limites du tableau (fig. 1) confrontent la littérature à la bande dessinée sur deux plans à la fois ${ }^{33}$ : non seulement 
le média visuel fournit ses thèmes au roman illustré (novellisation), mais il en influence encore l'écriture et l'illustration (rémanence).

Dans certains cas, des bribes d'intrigues issues d'une seule planche de BD se retrouvent au moins dans deux nouvelles différentes. Par exemple, dans le douzième gag ${ }^{34}$, le père de Nicolas, plongé dans son livre, l'autorise distraitement à inviter ses copains à la maison, par un jour pluvieux. Aussitôt, une bande de garnements envahit le salon, renversant un vase et blessant l'adulte avec une balle de football. De même, dans la nouvelle «Au chocolat et à la fraise»(HI1), Nicolas demande à ses parents l'autorisation d'inviter des amis, alors qu'il va pleuvoir. Sa mère semble se rappeler des circonstances du gag de bande dessinée: "La dernière fois qu'ils sont venus, tes copains, il a fallu remplacer deux carreaux de la fenêtre du salon et on a dû repeindre ta chambre. » (HI1, p. 499), à moins qu'elle ne se souvienne du "Vase rose du salon » (RPN) que l'enfant avait cassé en jouant seul au ballon. Dans une nouvelle antérieure intitulée « Louisette » (LPN), Nicolas faisait déjà référence à cet épisode :

Bien sûr, moi aussi je pleure quelquefois, mais c'est pour des choses graves, comme la fois où le vase du salon s'est cassé et que papa m'a grondé et ce n'était pas juste parce que je ne l'avais pas fait exprès et puis, ce vase, il était très laid et je sais bien que papa n'aime pas que je joue à la balle dans la maison, mais dehors, il pleuvait. (LPN, p. 80-82)

17 L'illustration du "Vase rose du salon » (RPN) présente un autre cas de réminiscence médiatique, puisque Nicolas y fait ses aveux dans une bulle compartimentée en douze images, décomposant graphiquement la trajectoire de la balle (fig. 2). Thierry Groensteen avait d'ailleurs retenu cette illustration dans Les Cahiers de la bande dessinée, lorsqu'il interrogeait Jean-Jacques Sempé sur la "forme narrative, ou séquentielle " $^{35}$ de ses dessins. En dépit de ses dénégations, l'illustrateur avait déjà fait la démonstration de cette hypothèse, dans les années 1950, en publiant un cartoon dans Moustique qui montrait le Petit Nicolas, la tête coincée dans le même vase rose ${ }^{36}$, puis en revisitant le gag, quelques années plus tard, en une séquence de six vignettes muettes (fig. 3), où Nicolas faisait semblant de s'être coincé la tête dans le vase, obligeant sa mère à le briser elle-même pour délivrer son fils farceur ${ }^{37}$. Comme le suggère le tableau (fig. 1), plusieurs des cartoons originaux ont pu inspirer les gags de BD avant d'être repris en récits littéraires. 


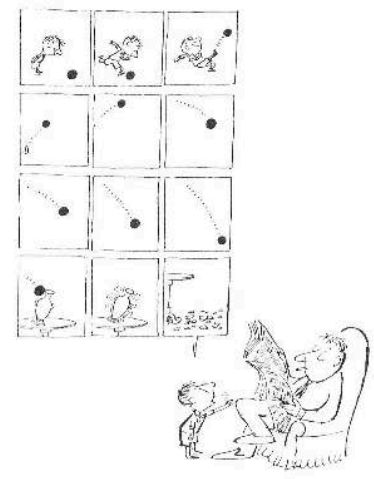

Ca, ça ne lui a pas plu, à Maman. "Ca, par exemple !" elle a dit, et puis elle est allée dans le salon.

- Alors, a dit Maman, c'est comme ça que tu fais l'éducation du petit?

Papa a levé la tête de son journal l'air très Papa
étonné.

Qu'est-ce que tu dis ? il a demandé.

- Ah! non, je t'en prie, ne fais pas l'innocent, a dit Maman. Evidemment, tu préfères lire tranquillement ton journal, pendant que moi je m'occupe de la discipline!

- J'aimerais en effet, a dit Papa, lire tranquillement mon journal, mais il semble que ce soit une chose impossible dans cette maison!

Fig. 2 : Rémanence de la bande dessinée dans l'illustration de roman. René Goscinny et Jean-Jacques Sempé, Les Récrés du Petit Nicolas, Paris, Gallimard, «Folio», 1994, p. 37. 2018, Éditions Gallimard / IMAV éditions / Goscinny - Sempé. D.R.

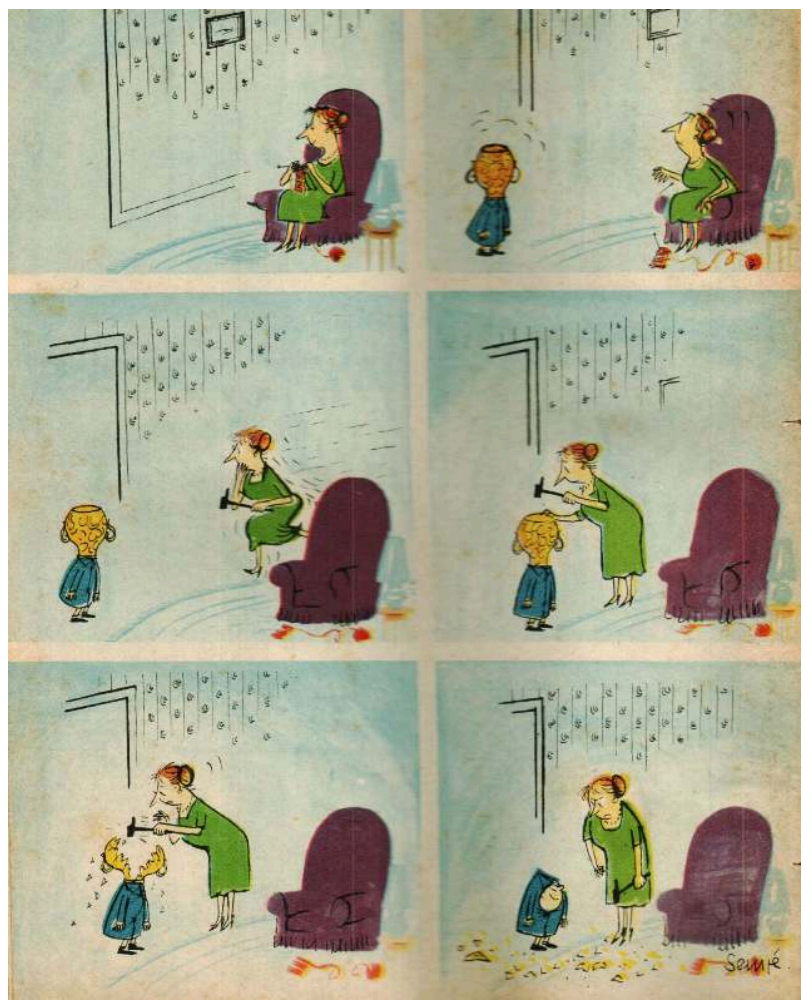

FIg. 3 : Rémanence de la BD dans le dessin de presse. Jean-Jacques Sempé, illustration de couverture sans titre, in TV Radio Moustique, n 1685, 11 mai 1958, Marcinelle, Dupuis, p. 80. 2018, IMAV éditions / Sempé. D.R. 


\section{Relire le Petit Nicolas comme une novellisation} son camarade de classe Nicolas, mais le père de ce dernier ne parvient pas à le faire fonctionner. La narration connaît six modifications importantes: transposition temporelle, re-focalisation, transvocalisation, expansion thématique, ajout d'un personnage et caractérisation des dialogues. Le récit passe d'une narration simultanée à une narration ultérieure des événements. Dans cet extrait, l'effet de style enfantin est rendu par un maniement maladroit des temps verbaux : le passé (sur)composé évolue vers un présent de vérité générale, celui du monologue intérieur.

«Si on mettait une des vaches là ?", j'ai demandé. «À qui il est le train, à toi ou à moi ?", a dit Alceste. Là, il avait raison, alors nous avons mis les vaches où avait décidé Alceste, qui a bien de la chance que le Père Noël lui fasse des cadeaux comme ça, parce qu'après tout, il n'a jamais été tellement sage, Alceste, et on le punit plus souvent que moi, et je suis moins mauvais élève que lui, et je suis beaucoup plus gentil qu'Alceste, et ce n'est pas juste, et je lui ai donné une claque. (HI1, p. 273) interne, par l'adoption du point de vue du Petit Nicolas. La narration à la première personne ne nécessite plus les soliloques livrés au lecteur dans la bande dessinée ${ }^{41}$. L'adresse au lecteur se fait désormais par des apartés, tels que «il faut vous dire qu'Alceste aime beaucoup manger " (HI1, p. 273). La dilatation des épisodes narratifs 
découle de l'ajout du personnage d'Alceste, qui modifie profondément le cadre participatif du dialogue. Sa rivalité avec le père de Nicolas, pour s'arroger le rôle de comparse du héros, se marque explicitement dans leurs impertinences réciproques. La novellisation introduit un autre épisode narratif, centré sur le père, en convertissant sa motivation initiale (« avoir la paix !» (v. 6)) en désir de jouer avec les enfants ${ }^{42}$ : «Papa a dit qu'il n'allait rien casser et qu'il allait nous montrer comment jouer au train électrique. [...] "Attention au départ! a crié papa avec une drôle de voix. En voituuuure! Tuuuuut!" (HI1, p. 275). Ainsi, le titre même de la nouvelle contrefait la voix de l'adulte se prenant au jeu.

Il existe enfin une adaptation pour la télévision, également intitulée Tuuuuut!, ainsi que sa novellisation sous le titre Papa casse mes jouets! Par rapport à la nouvelle de Sempé et Goscinny, la narration du roman connaît cinq nouveaux changements : transposition temporelle, re-focalisation, transvocalisation, expansion thématique et ajout d'épisodes narratif (justifiés par l'introduction de nouveaux personnages). La couverture montre Nicolas, dépité, tandis que son père joue sans lui avec un train électrique. Le titre Papa casse mes jouets! indique bien le passage du passé au présent de la narration, mais il introduit aussi deux confusions : d'une part, le papa de Nicolas n'y casse pas ses jouets (le train étant toujours à Alceste), d'autre part, la narration échappe à Nicolas, pour devenir hétérodiégétique et en focalisation zéro. Comme souvent dans les novellisations de film commerciales ${ }^{43}$, le roman crée de toute pièce une instance narrative extradiégétique, absente dans les versions précédentes du récit, y compris celles en bande dessinée et en dessin animé, qui ne recourent pas à la voixover. Pourtant, les novellisations de dessins animés pour la jeunesse - notamment les séries de romans Cédric, Titeuf et Yakari en "Bibliothèque rose " - ont institué le type de la " "novélisation confession", où l'énonciation se loge de façon spécifique dans le point de vue d'un protagoniste $»^{44}$, en s'inspirant vraisemblablement des nouvelles du Petit Nicolas.

Quant aux novellisations des dessins animés, elles mêlent une retranscription de la continuité dialoguée à une description du dessin animé, telle qu'un spectateur adulte pourrait la faire. Par exemple, cet extrait relate l'intrusion incongrue du voisin Blédurt dans la chambre de Nicolas :

Il surprend le papa de Nicolas, penché au-dessus du circuit, qui fait avancer le petit train à la main. « TCHOU-TCHOU ! » fait-il en mimant le bruit de la locomotive. M. Blédurt éclate de rire. «HA, HA, HA! HOU, HOU, HOU, HOU!» s'esclaffe-t-il, les larmes aux yeux. Qu'est-ce que tu as l'air ridicule à jouer au train comme un gamin ! 45

24 À nouveau, la novellisation introduit un effet de ridicule, censé provoquer un sentiment de honte, que René Goscinny n'avait pas explicité. Les redondances de cet extrait produisent une saturation informative qui interdit les antiphrases et les sousentendus. Les déclinaisons littéraires du Petit Nicolas illustrent deux façons opposées de traiter la parole dans l'écriture pour la jeunesse. Alors que les nouvelles originales laissent «s'exprimer un "je" qui se veut enfantin [...] par lequel l'adulte s'efforce explicitement de conquérir la confiance de celui qui le lit », les romans tirés des dessins animés donnent la parole « à ces adultes qui souhaitent assumer le rôle dominant de la communication $»^{46}$.

Un autre gag de bande dessinée, le septième ${ }^{47}$, repose sur une logique strictement visuelle : le père de Nicolas, pour lui apprendre à rouler sur le petit vélo qu'il vient de 
lui offrir, lui montre l'exemple en l'enfourchant lui-même. Le comique de situation est visuel, à la façon d'un cartoon: un adulte en costume-cravate s'arc-boutant sur un vélo trop petit pour lui (et y prenant manifestement du plaisir) s'oppose à l'enfant qui court derrière lui, à la place qu'il aurait dû occuper. Les dialogues de cette planche soulignent l'inversion des générations. Le discours du père évolue d'un propos adulte, mesuré et autoritaire, à un propos enfantin, égoïste et bêtifiant : "Regarde, Nicolas, je suis un train... tchou... tchou... tchou...» (v. 9). Les injonctions paternelles sont systématiquement transgressées par Nicolas. Au premier ordre, «Devine la surprise qui t'attend dehors. » (v. 2), le fils oppose des réponses farfelues (« Une vraie automobile? [etc.] »), il résiste également aux deux injonctions « Regarde ! / Cours derrière moi et tu verras comment je fais... » (v. 7-8), en s'arrêtant, sur le trottoir, pour jouer avec un camarade rencontré par hasard. Le dialogue des enfants singe une discussion de convenance entre adultes : «Tiens, mais c'est Nicolas... / J'ai des billes, Nicolas, on joue aux billes? ? / « Si tu as des billes, jouons aux billes, Timothée.» (v. 10-11). Le ridicule de la situation du père, roulant désormais seul sur un vélo d'enfant, est sanctionné par deux passantes âgées : «Pauvre garçon! / Quel malheur quand on devient gâteux !... » (v. 12). Le texte de cette planche saisit donc toutes les occasions de démultiplier l'inversion burlesque des générations, jouant la carte de la surenchère verbale.

Fidèles à la $\mathrm{BD}$, les dessins de Sempé pour la nouvelle «Le vélo" (LPN) suffisent à indiquer ce ressort burlesque. Le ridicule des scènes représentées nous invite à prendre le récit de Nicolas au second degré. La narration ultérieure « en je » suggère la forme d'un journal intime, ou bien celle d'une " composition française ", genre scolaire qui encourageait les élèves à dévoiler leur conception personnelle d'un événement passé ${ }^{48}$. Ce registre intime rend ambigu le statut des illustrations, loin de feindre un style graphique enfantin. Le lecteur doit « donc accepter la convention de cette juxtaposition d'une voix écrite et d'une image décrivant des actions énoncées simultanément ${ }^{49}$. Nicolas rapporte avec bienveillance les mots et les actes de son père :

Papa, il est resté avec moi dans le jardin. «Tu sais, il m'a dit, que j'étais un drôle de champion cycliste et que si je n'avais pas connu ta mère, je serais peut-être passé professionnel? » Ça, je ne le savais pas. Je savais que papa avait été un champion terrible de football, de rugby, de natation et de boxe, mais pour le vélo, c'était nouveau. "Je vais te montrer", a dit papa, et il s'est assis sur mon vélo et il a commencé à tourner dans le jardin. Bien sûr, le vélo était trop petit pour papa et il avait du mal avec ses genoux qui lui remontaient jusqu'à la figure, mais il se débrouillait. » (LPN, p. 120)

Certains des traits de style oralisé étaient déjà présents dans les gags de bande dessinée : la dislocation («Papa, il est resté avec moi »), l'abus des déictiques (« Ça, je ne le savais pas.», «c'était nouveau »), le lexique pauvre mais rarement familier («drôle de », «terrible»), les anacoluthes («le vélo était trop petit pour papa et il avait du mal») et les reprises, lexicales et syntaxiques. Bien entendu, d'autres traits littéraires ne proviennent pas de la BD, tels que les incises élémentaires («il m’a dit», «a dit papa »), les coq-à-l'âne digressifs, les discordances des temps et les usages dialogiques du discours rapporté, qui ont été analysés plus haut dans la nouvelle "Tuuuuut!». L'effet de monologue intérieur de la nouvelle est produit par les propositions brèves juxtaposées sans liaison (asyndètes) ou au contraire coordonnées alors qu'elles sont sans rapport fonctionnel (zeugmes). De cela même, on trouve la trace dans la séquencialité de la $\mathrm{BD}$, où les points de suspension et les sauts de bulles font office de connecteurs logiques entre des propositions brèves et souvent elliptiques. Remarquons, par ailleurs, que l'«idée de style " de René Goscinny, qui consistait à «inventer un 
langage de gosse $\aleph^{50}$, lui vint au moment où le roman Zazie dans le métro, paru à la mijanvier 1959, défrayait la chronique littéraire ${ }^{51}$.

Quant à la novellisation tirée du dessin animé correspondant, intitulée Papa m'offre un vélo, elle rend la scène précédente plus interactionnelle, en y introduisant la mère de Nicolas, quitte à retarder l'inversion burlesque qui était au cœur du gag de BD.

«Il faut que je te montre comment on fait.» Et il ajoute, tout fier : «Parce que, tu sais, si je n'avais pas rencontré ta mère, j'aurais pu être champion cycliste...» «Pardon?» sursaute la maman de Nicolas. «Bah, c'est vrai, j'étais en bonne position pour gagner le Tour de France, enfin... au moins, le concours régional », bafouille le papa de Nicolas. « Eh bien, ça fait plaisir à entendre! » lâche son épouse, vexée. Et elle retourne dans la maison, en claquant la porte derrière elle. Un quart d'heure plus tard, Nicolas n'a toujours pas réussi à approcher son vélo. Perché sur la petite selle, son papa fait des tours dans le jardin. ${ }^{52}$

Les voix énonciatives apparaissent cloisonnées: le style oralisé se cantonne aux dialogues, rapportés au discours direct, tandis que le style du narrateur dénote une correction langagière. Par exemple, les incises «il ajoute, tout fier ", "bafouille le papa ", "lâche son épouse, vexée» sont plus recherchées que dans la nouvelle de Sempé et Goscinny. Alors que toutes les autres versions superposaient les voix, ce qui perpétuait le dialogisme du gag de BD initial, la novellisation les cloisonne, en leur attribuant un ordre de succession. Les répétitions lexicales sont soigneusement évitées par l'emploi d'anaphores. Cet effort de correction grammaticale du texte original en clarifie et en dépersonnalise l'énonciation, ce qui lève les principales difficultés de lecture - «les répétitions » et "le mélange des paroles »- qu'il pose habituellement aux jeunes élèves (de $\mathrm{CM} 1$ et $\mathrm{CM} 2)^{53}$. Conçue comme une aide à la lecture, l'abondante iconographie, issue du dessin animé, privilégie les scènes d'interaction duratives (ce sont souvent des portraits de locuteurs, représentatifs d'une intervention verbale saillante), plutôt que les phases d'actions ponctuelles. L'image s'en trouve doublement " mise en sourdine », par la coupure de sa bande-son et par "la censure, de l'élément temporel et narratif des illustrations $»^{54}$. Ainsi, le dessin animé est tenu à distance du texte romanesque, tout comme le récit est mis à distance par la médiation d'un narrateur hétérodiégétique. Cette double distanciation produit l'impression de lire une description du dessin animé faite par un adulte pour un enfant, en cherchant à produire dans le texte - au contraire, paradoxalement, de l'illustration - un «effet d'image » donnant «l'impression que la scène se déroule sous les yeux du lecteur $»^{55}$.

\section{Conclusion}

Parmi toutes les œuvres du réseau intermédiatique du Petit Nicolas, seules les nouvelles parues entre 1959 et 1965 ont passé à la postérité et elles ont le moins de chances d'être perçues comme des (auto)adaptations. Aujourd'hui, grâce à leurs qualités littéraires, ces récits illustrés qui «[ont] révolutionn[é] le livre conçu pour le jeune public en adoptant le rythme de la narration à celui de la conversation $»^{56}$ constituent des récits médiagéniques, dans la mesure où ils sont devenus des " œuvres sources ", éclipsant les hypotextes dont ils s'étaient nourris, et donnant l'impression d'avoir définitivement adopté le média littéraire dans lequel ils se sont optimalement déployés. En fait, l'évaluation $d u$ " quotient d'adaptabilité ${ }^{57}$ d'une œuvre nous invite non pas à récuser, mais bien à historiciser, le sentiment d'une médiagénie. Dans les années 1960, cette médiagénie des récits des Récrés du Petit Nicolas était confirmée par leur universalité, si 
l'on retient la formule de Sempé, «c'est un livre pour les parents quand ils ont des enfants et pour les enfants quand ils ont des parents $\|^{58}$. Ce phénomène de double adresse, cependant, n'est pas une propriété immanente de l'«œuvre source ", il découle de son histoire éditoriale, à commencer par sa naissance dans des périodiques (Le Moustique, Sud-Ouest Dimanche, Pilote) qui ne visaient pas prioritairement les enfants. Cet exemple montre que la notion de médiagénie ne restaure pas une vision téléologique de l'adaptation. Au contraire, les nouvelles illustrées du Petit Nicolas défient la vision linéaire qui sous-tend les études d'adaptation traditionnelles, car chacune de leurs variations médiatiques, de 1954 à nos jours, "constitue une adaptation créative et interprétative du texte source, participant ainsi, en tant qu'intertextes visuels, à l'établissement sans fin du texte source en tant que palimpseste $\aleph^{59}$.

31 Le remplacement du problème de fidélité par la question d'une circulation intermédiatique, suivant des logiques de reprise et de variation, s'applique non seulement aux nouvelles, dont les bandes dessinées sources sont restées longtemps inaccessibles, mais aussi aux novellisations actuelles de la série d'animation télévisée. Si la majorité des dessins animés adaptent des nouvelles de Sempé et Goscinny, dont ils adoptent les titres, ils sont probablement perçus par leur public enfantin davantage comme des continuations sérialisées d'un univers fictionnel, que comme les adaptations qu'ils sont effectivement. Leur novellisation ne se justifie pas seulement par une motivation commerciale, dans le cadre de la promotion de la série d'animation, mais aussi par une question de survie par la transmédiatisation, dès lors que la lecture des nouvelles originales pose des difficultés aux jeunes lecteurs des années 2000 . Le public enfantin visé par les romans d'Emmanuelle Lepetit ne s'inquiète pas de leur fidélité aux textes de René Goscinny, d'autant plus qu'il n'a aucune chance d'identifier la signature de la novellisatrice. Non seulement son nom n'est pas attaché à une œuvre (re)connue, mais en plus il n'apparaît pas sur la couverture des novellisations. Le processus novellisatoire, à peine plus explicite sur ces romans des années 2000 que sur les nouvelles des années 1960, n'en est pas moins perçu comme créateur d'inédit. En somme, ces multiples novellisations permettent à l'œuvre de survivre (au sens de " maintenir un changement constant, et ce à travers les trois opérations imbriquées de l'introduction du neuf, du passage à la série et de l'adaptation intermédiatique $\left.{ }^{60}\right)$. La novellisation dans le réseau intermédiatique du Petit Nicolas apparaît moins comme un procédé d'adaptation que de continuation. Ce processus créatif indissociable d'une pratique culturelle préfigure une conception très actuelle de la «novélisation » pour la jeunesse : «c'est parce que les éditeurs [tels qu'Hachette Jeunesse] misent d'abord sur un effet de continuum entre supports audiovisuel et textuel plutôt que sur des effets d'écarts et de réécriture au sens fort que la novélisation connaît un essor considérable dans les années $2000 »^{61}$. 


\section{NOTES}

1. Armelle Blin-Rolland, «Fidelity versus appropriation in comics adaptation: Jacques Carelman's and Clément Oubrerie's Zazie dans le métro ", European Comic Art, 6, 1, 2013, pp. 92-93.

2. Jan Baetens, "L'adaptation, une stratégie d'écrivain?", dans Benoît Mitaine, David Roche et Isabelle Schmitt-Pitiot (dir.), Bande dessinée et adaptation (littérature, cinéma, TV), ClermontFerrand, Presses universitaires Blaise-Pascal, « Littératures », 2015, p. 45.

3. Philippe Marion, «Emprise graphique et jeu de l'oie. Fragments d'une poétique de la bande dessinée ", dans Éric Maigret et Matteo Stefanelli (dir.), La Bande dessinée : une médiaculture, Paris, Armand Colin - INA, « Médiacultures », 2012, p. 190.

4. Jean-Louis Jeannelle, « Réadaptation », Critique, 795-796, 2013, p. 622.

5. Michel Melot, L'Gil qui rit. Le pouvoir comique des images, Fribourg, Office du livre, 1975, p. 27.

6. René Goscinny et Jean-Jacques Sempé, Le Petit Nicolas. La bande dessinée originale, Paris, IMAV, 2017, 47 p.

7. José-Louis Bocquet, Goscinny et moi. Témoignages, Paris, Flammarion, «Pop culture », 2007, pp. 106, 109 ; Jacques Glénat et Numa Sadoul, «Entretien avec René Goscinny », Les Cahiers de la bande dessinée, 22, 1973, p. 14.

8. Jan Baetens, «L'adaptation, une stratégie d'écrivain? », op. cit., p. 47.

9. Moustique, $\mathrm{n}^{\circ} 1562,1^{\mathrm{er}}$ janvier 1956, p. 13.

10. Bertrand et Christelle Pissavy-Yvernault, Franquin, Morris, Jijé, Sempé... 200 couvertures inédites pour le journal Le Moustique, Marcinelle, Dupuis, 2015, p. 230.

11. Moustique, $\mathrm{n}^{\circ} 1530,22$ mai 1955, p. 2.

12. Jérôme Dupuis, «Les aventures du Petit Nicolas racontées par Sempé », Lire - Le Vif - L'Express, hors-série : La vie secrète de Goscinny, 2007, p. 54. Voir aussi : José-Louis Bocquet, Goscinny et moi, op. cit., pp. 103-104.

13. Voir la plupart d'entre elles dans Bertrand et Christelle Pissavy-Yvernault, Franquin, Morris, Jijé, Sempé... 200 couvertures inédites pour le journal Le Moustique, op. cit., pp. 231-233, 250-252, 257, 261 et 284.

14. Plus une ultime le 8 décembre 1957 (Moustique, $n^{\circ} 1663$ ).

15. Moustique, $\mathrm{n}^{\circ} 1488,1^{\mathrm{er}}$ août 1954, p. 2.

16. René Henoumont, Des amours de papier. Mémoires impertinentes, Louvain-la-Neuve, Duculot, « Documents témoignages », 1990, p. 205.

17. Les planches du Petit Nicolas occuperont effectivement l'espace stratégique de la dernière page du magazine (p. 80), en couleurs ou en gris (pour deux gags), presque chaque semaine d'août 1955 à mai 1956, mais on trouvera aussi trois gags en bichromie à l'intérieur du magazine (p. 17).

18. Moustique, $\mathrm{n}^{\circ} 1562,1^{\mathrm{er}}$ janvier 1956, p. 12 .

19. Moustique, $\mathrm{n}^{\circ} 1558,4$ décembre 1955, p. 3.

20. Sempé prolongea l'un de ses gags de BD dans une couverture, en reprenant une idée de Franquin (Moustique, $\mathrm{n}^{\circ} 1349,1558,1663$ ), et il publia surtout une planche muette qui développait un cartoon du Petit Nicolas ( $\left.n^{\circ} 1493,1685\right)$; l'illustrateur Benoist (Benoît Gillain) en adapta un autre en couverture $\left(\mathrm{n}^{\circ} 1488,1639\right)$; Morris réalisa une couverture répondant à une autre du Petit Nicolas $\left(n^{\circ} 1510,1611\right)$

21. Jacques Glénat et Numa Sadoul, « Entretien avec René Goscinny », op. cit., pp. 13-14.

22. Le vingt-huitième gag (Moustique, $\mathrm{n}^{\circ}$ 1582, 20 mai 1956, p. 80) devait encore participer au lancement du Supplément illustré, qui ne connut qu'un numéro Pilote en 1956, sous la houlette de l'agence Édifrance-Édipresse fondée notamment par Goscinny. 
23. José-Louis Bocquet, Goscinny et moi, op. cit., p. 104. Curieusement, le rédacteur en chef du Moustique, alors qu'il assume l'initiative de la commande des planches à Sempé, exprime les mêmes réticences que lui envers le média : «Pas drôle, d'être dessinateur de bandes dessinées! En ce qui me concerne, le caractère répétitif de leurs histoires me lassait, quelle que fût l'envolée du coup de crayon. Depuis ma prime enfance j'étais un homme de textes, et leur parcimonie dans les petits ballons me laissait sur ma faim » (René Henoumont, Des amours de papier, op. cit., p. 128). 24. Thierry Groensteen, "Entretien avec Sempé », Les Cahiers de la bande dessinée, 78, 1987, pp. 54-55. Dans un contexte de légitimation du dessin d'humour, ses défenseurs se livrent à une chasse aux bulles dans le cartoon, qui implique parfois un dénigrement de la BD. Par exemple, Michel Melot considère Jean-Jacques Sempé comme un spécialiste du «genre de l'image en plusieurs tableaux ", qu'il distingue résolument de la bande dessinée, en replaçant plutôt dans la lignée de Chaval et de Jean Bosc «cette alliance contre nature entre le spectacle, qui est évolution, et l'image, qui est fixation » (Michel Melot, L'ÆEil qui rit, op. cit., pp. 146-148).

25. José-Louis Bocquet, Goscinny et moi, op. cit., p. 105.

26. Jérôme Dupuis, « Les aventures du Petit Nicolas racontées par Sempé », op. cit., p. 56.

27. Des deux films en live-action, seul le second a connu une novellisation, par Valérie LatourBurney, mais le format de ce long métrage ne supporte pas la comparaison avec les œuvres brèves du tableau.

28. Les éditions citées seront les suivantes : Le Petit Nicolas, Paris, Gallimard, «Folio ", 1975 ; Les Récrés du Petit Nicolas, Paris, Gallimard, «Folio », 1994; Histoires inédites du Petit Nicolas, Paris, IMAV, vol. 1, 2004 ; vol. 2, 2006.

29. René Goscinny et Jean-Jacques Sempé, Le Petit Nicolas. La bande dessinée originale, op. cit., p. 22.

30. Pierre Desgraupes, René Goscinny et Jean-Jacques Sempé, «Lectures pour tous : Sempé et Goscinny à propos du livre Les Vacances du Petit Nicolas », ORTF, $1^{\mathrm{er}}$ août 1962, en ligne, http:// www.ina.fr/video/I08162779 (page consultée le 3 novembre 2017).

31. À l'inverse, dans la nouvelle « Djodjo » $(L P N)$, les trois insultes apprises par l'élève étranger apparaissent à la fois dans le texte et dans l'illustration, également à l'intérieur d'une bulle.

32. Philippe Marion, « Emprise graphique et jeu de l'oie », op. cit., p. 188.

33. Matthieu Letourneux, "Littérature de jeunesse et culture médiatique », dans Nathalie Prince (dir.), La Littérature de jeunesse en question(s), Rennes, Presses universitaires de Rennes, «Interférences », 2009, p. 202.

34. René Goscinny et Jean-Jacques Sempé, Le Petit Nicolas. La bande dessinée originale, op. cit., p. 16.

35. Thierry Groensteen, « Entretien avec Sempé », op. cit., pp. 55-56.

36. Moustique, $\mathrm{n}^{\circ}$ 1493, 5 septembre 1954, p. 11.

37. TV Radio Moustique, $\mathrm{n}^{\circ}$ 1685, 11 mai 1958, p. 80. Et ce gag sera recyclé dans la nouvelle « Un souvenir qu'on va chérir » (LPN), où, pendant une séance de photo de classe, Geoffroy se coince la tête dans son casque de déguisement.

38. Quelques personnages enfantins apparaissent sporadiquement: un petit Timothée (gag $n^{\circ} 7$ ), un certain Jocelyn (gag $n^{\circ} 21$ ) et d'autres anonymes (gags $n^{\circ} 2,12$ et 20 ). Nicolas joue plus longuement avec un petit Alceste ( $\left.g a g n^{\circ} 16\right)$, qui ne ressemble pas à son futur comparse, et avec

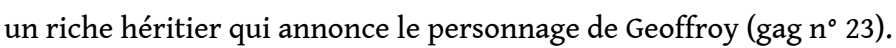

39. Benoît Glaude, «La novellisation des dialogues de bande dessinée », Image \& Narrative, 17, 3, 2016, pp. 14-18.

40. Moustique, $\mathrm{n}^{\circ}$ 1558, 4 décembre 1955, p. 80. René Goscinny et Jean-Jacques Sempé, Le Petit Nicolas. La bande dessinée originale, op. cit., p. 15.

41. Les nombreux soliloques du gag de $\mathrm{BD}$, qui clarifient les intentions du père de Nicolas, apparaissent dans des bulles de dialogues (et non de pensées), mais ils ne reçoivent pas de réponse dans la diégèse, parce qu'ils s'adressent plus au lecteur qu'à Nicolas.

42. Ce gag supplémentaire sera repris par Sempé pour une couverture du Moustique ( ${ }^{\circ} 1663,8$ décembre 1957). Six ans plus tôt, la même idée se trouvait dans une couverture ( $n^{\circ} 1349,2$ 
décembre 1951) d'André Franquin, où un père constate que son jouet électrique ne fonctionne plus : « Fiston, je suis sûr que tu as encore touché à ce train ! ».

43. Jan Baetens, La Novellisation. Du film au roman. Lectures et analyses d'un genre hybride, Bruxelles, Les Impressions Nouvelles, « Réflexions faites », 2008, p. 75.

44. Prisca Grignon, Le Champ d'existence de la novélisation francophone actuelle (10 dernières années). La novélisation, un nouveau genre ?, thèse de doctorat en études cinématographiques, Université de Montpellier, 2012, p. 109.

45. Emmanuelle Lepetit, Le Petit Nicolas, t. 19: Papa casse mes jouets!, Paris, Gallimard, «Folio cadet. Premières lectures ", 2014, pp. 12-13.

46. Jean Perrot, «Parler comme un livre », dans Du jeu, des enfants et des livres, Paris, Cercle de la librairie, «Bibliothèques », 1987, p. 227. Voir aussi : Ganna Ottevaere-van Praag, «Le dialogue dans les livres français pour la jeunesse. Histoire succincte d'une adaptation linguistique », Linguistica Antwerpiensia, 16-17, 1983, p. 194.

47. Moustique, $\mathrm{n}^{\circ}$ 1554, 6 novembre 1955, p. 80. René Goscinny et Jean-Jacques Sempé, Le Petit Nicolas. La bande dessinée originale, op. cit., p. 34.

48. Jean Perrot a mis en évidence, dans l'incipit des nouvelles du Petit Nicolas, une exposition du thème typique de l'introduction d'une composition française (Jean Perrot, "Parler comme un livre», op.cit., pp. 252-254). Au contraire, les autres versions des récits - les BD, les dessins animés et leurs novellisations - commencent toujours in medias res.

49. Jean Perrot, « Parler comme un livre », op. cit., p. 258.

50. Jacques Glénat et Numa Sadoul, « Entretien avec René Goscinny », op. cit., p. 14.

51. Cependant, le style oralisé des nouvelles du Petit Nicolas diffère de celui de Zazie dans le métro au moins sur trois points : « les discordantiels sont presque toujours présents, les indicateurs de style direct ont en général la forme la plus simple qui soit ( $X$ a dit), et l'orthographe n'est presque jamais phonétisée » (Daniel et Françoise Luzzati, «Oral et familier : le style oralisé », L'Information grammaticale, 34, 1987, p. 17).

52. Emmanuelle Lepetit, Le Petit Nicolas, t. 4 : Papa m'offre un vélo, Paris, Gallimard, «Folio cadet. Premières lectures ", 2012, pp. 8-10.

53. Claude Le Manchec et Joël Manchon, «Une lecture à voix haute peut-elle favoriser la découverte des enjeux de l'instance narrative du Petit Nicolas? ", dans Philippe Clermont et Anne Schneider (dir.), Écoute mon papyrus. Littérature, oral et oralité, Strasbourg, CRDP d'Alsace, « Documents, actes et rapports pour l'éducation », 2006, p. 199.

54. Jan Baetens, La Novellisation, op. cit., p. 91.

55. Matthieu Letourneux, «Littérature de jeunesse et culture médiatique », op. cit., p. 198.

56. Ganna Ottevaere-van Praag, «Le dialogue dans les livres français pour la jeunesse », op. cit., pp. $185-186$.

57. Jean-Louis Jeannelle, « Réadaptation », op. cit., p. 620.

58. Pierre Dumayet, René Goscinny et Jean-Jacques Sempé, «Lectures pour tous: Sempé et Goscinny à propos du livre Les Récrés du Petit Nicolas ", ORTF, 13 décembre 1961, en ligne, http:// www.ina.fr/video/I05312382 (page consultée le 3 novembre 2017).

59. Armelle Blin-Rolland, « Fidelity versus appropriation in comics adaptation », op. cit., p. 108 (je traduis).

60. Jan Baetens, La Novellisation, op. cit., p. 75.

61. Prisca Grignon, Le Champ d'existence de la novélisation francophone actuelle, op. cit., p. 123. 


\section{ABSTRACTS}

The intermedial network of Little Nicholas is not limited to Jean-Jacques Sempé's and René Goscinny's illustrated short stories (1959-1965), which have become classics of children's literature. Before that, they were born in cartoons and comics, and they have been brought to the big and small screen ever since, including an animated TV series, which is being novelized. This paper proposes a reading of some Little Nicholas' short stories as direct novelizations - and some novels based on animated cartoons, as indirect novelizations - of the comics pages published in 1955-1956. Novelization will be considered both as a cultural practice and as a creative process.

Le réseau intermédiatique du Petit Nicolas ne se réduit pas aux nouvelles illustrées de JeanJacques Sempé et René Goscinny (1959-1965), devenues des classiques de la littérature pour la jeunesse. L'œuvre est née dans des cartoons et des bandes dessinées et récemment elle a investi le grand et le petit écran, notamment dans une série d'animation télévisée toujours en cours de novellisation. Cet article propose de relire quelques nouvelles du Petit Nicolas comme des novellisations directes (et quelques romans tirés des dessins animés, comme des novellisations indirectes) des gags de BD parus au milieu des années 1950. La novellisation est ici considérée à la fois comme une pratique culturelle et comme un processus créatif.

\section{INDEX}

Mots-clés: bande dessinée, novellisation, adaptation, littérature pour la jeunesse, intermédialité

\section{AUTHOR}

\section{BENOÎT GLAUDE}

FNRS / Université catholique de Louvain 